Annals of Pure and Applied Mathematics

Vol. 20, No. 1, 2019, 1-8

ISSN: 2279-087X (P), 2279-0888(online)

Published on 26 June 2019

Annals of

www.researchmathsci.org

DOI: http://dx.doi.org/10.22457/apam.622v20n1a1

Pure and Applied

Mathematics

\title{
New Connectivity Topological Indices
}

\author{
V.R.Kulli
}

Department of Mathematics

Gulbarga University, Gulbarga 585106, India

e-mail: vrkulli@gmail.com

Received 1 June 2019; accepted 25 June 2019

Abstract. New degree based graph indices called Kulli-Basava indices were introduced and studied their mathematical and chemical properties which have good response with mean isomer degeneracy. In this study, we introduce the sum connectivity Kulli-Basava index, product connectivity Kulli-Basava index, atom bond connectivity Kulli-Basava index and geometric-arithmetic Kulli-Basava index of a graph and compute exact formulas for some special graphs.

Keywords: Connectivity Kulli-Basava indices, wheel, gear, helm graphs

AMS Mathematics Subject Classification (2010): 05C05, 05C07, 05C12, $05 C 35$

\section{Introduction}

Let $G$ be a finite, simple connected graph with vertex set $V(G)$ and edge set $E(G)$. The degree of $d_{G}(v)$ of a vertex $v$ is the number of vertices adjacent to $v$. The degree of an edge $e=u v$ in $G$ is defined by $d_{G}(e)=d_{G}(u)+d_{G}(v)-2$. The open neighborhood $N_{G}(v)$ of a vertex $v$ is the set of all vertices adjacent to $v$. The edge neighborhood of a vertex $v$ is the set of all edges incident to $v$ and it is denoted by $N_{e}(v)$. Let $S_{e}(v)$ denote the sum of the degrees of all edges incident to a vertex $v$. We refer to [1] for undefined term and notation.

A topological index is a numerical parameter mathematically derived from the graph structure. Several topological indices have been considered in Theoretical Chemistry, see [2, 3].

The first and second Kulli-Basava indices were introduced in [4], defined as

$$
\begin{aligned}
& K B_{1}(G)=\sum_{u v \in E(G)}\left[S_{e}(u)+S_{e}(v)\right], \\
& K B_{2}(G)=\sum_{u v E(G)} S_{e}(u) S_{e}(v) .
\end{aligned}
$$

The first and second hyper Kulli-Basava indices were introduced by Kulli [5], defined as

$$
H K B_{1}(G)=\sum_{u v E(G)}\left[S_{e}(u)+S_{e}(v)\right]^{2}, \quad H K B_{2}(G)=\sum_{u v E(G)}\left[S_{e}(u) S_{e}(v)\right]^{2} .
$$


V.R.Kulli

We introduce the sum connectivity Kulli-Basava index, product connectivity Kulli-Basava index, atom bond connectivity Kulli-Basava index, geometric-arithmetic Kulli-Basava index and reciprocal Kulli-Basava index of a graph, defined as

$$
\begin{aligned}
& S K B(G)=\sum_{u v \in E(G)} \frac{1}{\sqrt{S_{e}(u)+S_{e}(v)}}, \\
& P K B(G)=\sum_{u v \in E(G)} \frac{1}{\sqrt{S_{e}(u) S_{e}(v)}}, \\
& A B C K B(G)=\sum_{u v \in E(G)} \sqrt{\frac{S_{e}(u)+S_{e}(v)-2}{S_{e}(u) S_{e}(v)}} \\
& G A K B(G)=\sum_{u v \in E(G)} \frac{2 \sqrt{S_{e}(u) S_{e}(v)}}{S_{e}(u)+S_{e}(v)}, \\
& R K B(G)=\sum_{u v \in E(G)} \sqrt{S_{e}(u) S_{e}(v)} .
\end{aligned}
$$

Recently, some connectivity indices were studied $[6,7,8,9,10]$. In this paper, some connectivity Kulli-Basava indices for some graphs were computed.

\section{Regular graphs}

A graph $G$ is $r$-regular if the degree of every vertex of $G$ is $r$.

Theorem 1. Let $G$ be an $r$-regular graph with $n$ vertices and $m$ edges. Then

(i)

$$
\begin{aligned}
& S K B(G)=\frac{m}{2 \sqrt{r(r-1)}} . \\
& A B C K B(G)=\frac{m \sqrt{4 r(r-1)-2}}{2 r(r-1)} .
\end{aligned}
$$

(ii) $\operatorname{PKB}(G)=\frac{m}{2 r(r-1)}$.

(v)

$$
R K B(G)=2 m r(r-1) \text {. }
$$

Proof: Let $G$ be an $r$-regular graph with $n$ vertices and $m$ edges. Then $S_{e}(u)=2 r(r-1)$ for any vertex $u \in V(G)$. Therefore

$$
S K B(G)=\sum_{u v \in E(G)} \frac{1}{\sqrt{S_{e}(u)+S_{e}(v)}}=\frac{m}{\sqrt{2 r(r-1)+2 r(r-1)}}=\frac{m}{2 \sqrt{r(r-1)}} .
$$

$$
P K B(G)=\sum_{u v \in E(G)} \frac{1}{\sqrt{S_{e}(u) S_{e}(v)}}=\frac{m}{\sqrt{2 r(r-1) 2 r(r-1)}}=\frac{m}{2 r(r-1)} .
$$

$$
\begin{aligned}
A B C K B(G) & =\sum_{u v \in E(G)} \sqrt{\frac{S_{e}(u)+S_{e}(v)-2}{S_{e}(u) S_{e}(v)}}=\frac{m \sqrt{2 r(r-1)+2 r(r-1)-2}}{\sqrt{2 r(r-1) 2 r(r-1)}} \\
& =\frac{m \sqrt{4 r(r-1)-2}}{2 r(r-1)} .
\end{aligned}
$$


New Connectivity Topological Indices

(iv) $\quad G A K B(G)=\sum_{u v \in E(G)} \frac{2 \sqrt{S_{e}(u) S_{e}(v)}}{S_{e}(u)+S_{e}(v)}=\frac{m 2 \sqrt{2 r(r-1) 2 r(r-1)}}{2 r(r-1)+2 r(r-1)}=m$.
(v) $R K B(G)=\sum_{u v \in E(G)} \sqrt{S_{e}(u) S_{e}(v)}=m \sqrt{2 r(r-1) 2 r(r-1)}=2 m r(r-1)$.

Corollary 1.1. If $C_{n}$ is a cycle with $n$ vertices, then

(i) $\quad \operatorname{SKB}\left(C_{n}\right)=\frac{n}{2 \sqrt{2}}$.

(ii) $\operatorname{PKB}\left(C_{n}\right)=\frac{n}{4}$.

(iii)
$A B C K B\left(C_{n}\right)=\frac{\sqrt{6}}{4} n$.
(iv) $\operatorname{GAKB}\left(C_{n}\right)=n$.

(v) $\quad R K B\left(C_{n}\right)=4 n$.

Corollary 1.2. If $K_{n}$ is a complete graph with $n$ vertices, then

$$
\begin{array}{ll}
\operatorname{SKB}\left(K_{n}\right)=\frac{n \sqrt{n-1}}{4 \sqrt{n-2}} . & \text { (ii) } \operatorname{SKB}\left(K_{n}\right)=\frac{n}{4(n-2)} . \\
A B C K B\left(K_{n}\right)=\frac{n \sqrt{4(n-1)(n-2)-2}}{4(n-2)} & \text { (iv) } \operatorname{GAKB}\left(K_{n}\right)=\frac{n(n-1)}{2} .
\end{array}
$$

$$
R K B\left(K_{n}\right)=n(n-1)^{2}(n-2) \text {. }
$$

\section{Results for wheel graphs}

A wheel $W_{n}$ is the join of $C_{n}$ and $\mathrm{K}_{1}$. Clearly $W_{n}$ has $n+1$ vertices and $2 n$ edges. A wheel $W_{n}$ is shown in Figure 1. The vertices $C_{n}$ are called rim vertices and the vertex of $K_{1}$ is called apex.

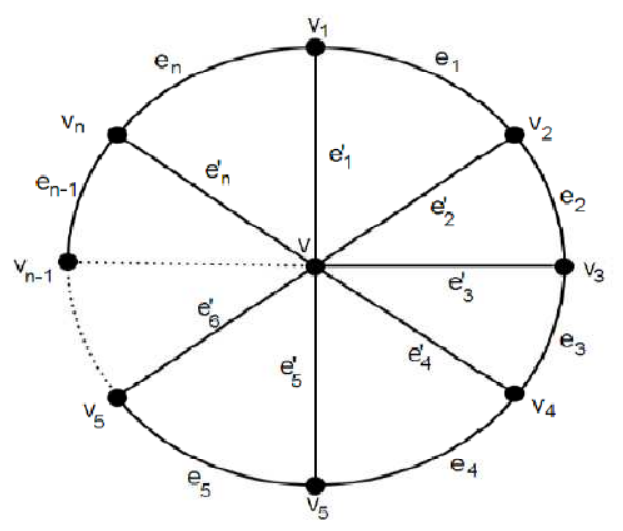

Figure 1: Wheel $W_{n}$

Lemma 2. Let $W_{n}$ be a wheel with $2 n$ edges, $n \geq 3$. Then

$$
\begin{array}{ll}
E_{1}=\left\{u v \in E\left(W_{n}\right) \mid S_{e}(u)=n(n+1), S_{e}(v)=n+9\right\}, & \left|E_{1}\right|=n . \\
E_{2}=\left\{u v \in E\left(W_{n}\right) \mid S_{e}(u)=S_{e}(v)=n+9\right\}, & \left|E_{2}\right|=n .
\end{array}
$$


Theorem 3. Let $W_{n}$ be a wheel with $n+1$ vertices and $2 n$ edges, $n \geq 3$. Then

(i) $\quad \operatorname{SKB}\left(W_{n}\right)=\frac{n}{\sqrt{n^{2}+2 n+9}}+\frac{n}{\sqrt{2 n+18}}$.

(ii) $\quad \operatorname{PKB}\left(W_{n}\right)=\frac{n}{\sqrt{n(n+1)(n+9)}}+\frac{n}{n+9}$.

(iii) $\quad A B C K B\left(W_{n}\right)=n \sqrt{\frac{n^{2}+2 n+7}{n(n+1)(n+9)}}+\frac{n \sqrt{2 n+16}}{n+9}$.

(iv) $\quad G A K B\left(W_{n}\right)=\frac{2 n \sqrt{n(n+1)(n+9)}}{n^{2}+2 n+9}+n$.

(v) $R K B\left(W_{n}\right)=n \sqrt{n^{2}+2 n+9}+n(n+9)$.

Proof: Let $W_{n}$ be a wheel with $n+1$ vertices and $2 n$ edges. By using definitions and Lemma 2, we obtain

(i) $\quad S K B\left(W_{n}\right)=\sum_{u v \in E\left(W_{n}\right)} \frac{1}{\sqrt{S_{e}(u)+S_{e}(v)}}=\frac{n}{\sqrt{n(n+1)+n+9}}+\frac{n}{\sqrt{n+9+n+9}}$

$$
=\frac{n}{\sqrt{n^{2}+2 n+9}}+\frac{n}{\sqrt{2 n+18}} \text {. }
$$

(ii) $\quad P K B\left(W_{n}\right)=\sum_{u v \in E\left(W_{n}\right)} \frac{1}{\sqrt{S_{e}(u) S_{e}(v)}}=\frac{n}{\sqrt{n(n+1)(n+9)}}+\frac{n}{\sqrt{(n+9)(n+9)}}$

$$
=\frac{n}{\sqrt{n(n+1)(n+9)}}+\frac{n}{n+9} \text {. }
$$

(iii) $\quad A B C K B\left(W_{n}\right)=\sum_{u v \in E\left(W_{n}\right)} \sqrt{\frac{S_{e}(u)+S_{e}(v)-2}{S_{e}(u) S_{e}(v)}}$

$$
\begin{aligned}
& =n \sqrt{\frac{n(n+1)+n+9-2}{n(n+1)(n+9)}}+n \sqrt{\frac{n+9+n+9-2}{(n+1)(n+9)}} \\
& =n \sqrt{\frac{n^{2}+2 n+7}{n(n+1)(n+9)}}+n \frac{\sqrt{2 n+16}}{(n+9)} .
\end{aligned}
$$

(iv) $\quad \operatorname{GAKB}\left(W_{n}\right)=\sum_{u v E\left(W_{n}\right)} \frac{2 \sqrt{S_{e}(u) S_{e}(v)}}{S_{e}(u)+S_{e}(v)}=\frac{2 n \sqrt{n(n+1)(n+9)}}{n(n+1)+(n+9)}+\frac{2 n \sqrt{(n+9)(n+9)}}{(n+9)+(n+9)}$

$$
=\frac{2 n \sqrt{n(n+1)(n+9)}}{n^{2}+2 n+9}+n \text {. }
$$

(v) $\quad R K B\left(W_{n}\right)=\sum_{u v \in E\left(W_{n}\right)} \sqrt{S_{e}(u) S_{e}(v)}=n \sqrt{n(n+1)(n+9)}+n \sqrt{(n+9)(n+9)}$

$$
=n \sqrt{n^{2}+2 n+9}+n(n+9) \text {. }
$$




\section{New Connectivity Topological Indices}

\section{Results for gear graphs}

A gear graph is obtained from $W_{n}$ by adding a vertex between each pair of adjacent rim vertices and it is denoted by $G_{n}$. Clearly $G_{n}$ has $2 n+1$ vertices and $3 n$ edges. A graph $G_{n}$ is shown in Figure 2.

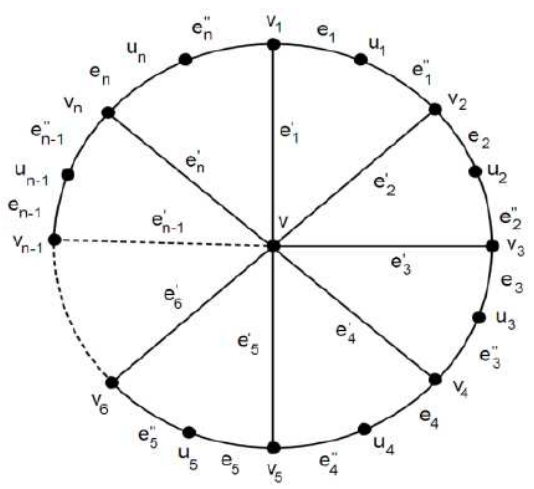

Figure 2: A gear graph $G_{n}$

Lemma 4. Let $G_{n}$ be a gear graph with $2 n+1$ vertices and $3 n$ edges. Then $G_{n}$ has two types of edges as follows:

$$
\begin{array}{ll}
E_{1}=\left\{u v \in E\left(G_{n}\right) \mid S_{e}(u)=n(n+1), S_{e}(v)=n+7\right\}, & \left|E_{1}\right|=n . \\
E_{2}=\left\{u v \in E\left(G_{n}\right) \mid S_{e}(u)=n+7, S_{e}(v)=6\right\}, & \left|E_{2}\right|=2 n .
\end{array}
$$

Theorem 5. Let $G_{n}$ be a gear graph with $2 n+1$ vertices $3 n$ edges. Then

(i) $\quad \operatorname{SKB}\left(G_{n}\right)=\frac{n}{\sqrt{n^{2}+2 n+7}}+\frac{2 n}{\sqrt{n+13}}$.

(ii) $\quad P K B\left(G_{n}\right)=\frac{n}{\sqrt{n(n+1)(n+9)}}+\frac{2 n}{\sqrt{6(n+7)}}$.

(iii) $\quad A B C K B\left(G_{n}\right)=n\left(\frac{n^{2}+2 n+5}{n(n+1)(n+7)}\right)^{\frac{1}{2}}+2 n\left(\frac{n+11}{6(n+7)}\right)^{\frac{1}{2}}$.

(iv) $\quad \operatorname{GAKB}\left(G_{n}\right)=\frac{2 n \sqrt{n(n+1)(n+7)}}{n^{2}+2 n+7}+\frac{4 n \sqrt{6(n+7)}}{n+13}$.

(v) $\quad R K B\left(G_{n}\right)=n \sqrt{n(n+1)(n+7)}+2 n \sqrt{6(n+7)}$.

Proof: Let $G_{n}$ be a gear graph with $2 n+1$ vertices and $3 n$ edges. By using definitions and Lemma 4, we obtain

(i) $\quad S K B\left(G_{n}\right)=\sum_{u v \in E\left(G_{n}\right)} \frac{1}{\sqrt{S_{e}(u)+S_{e}(v)}}=\frac{n}{\sqrt{n(n+1)+(n+7)}}+\frac{2 n}{\sqrt{n+7+6}}$

$$
=\frac{n}{\sqrt{n^{2}+2 n+7}}+\frac{2 n}{\sqrt{n+13}} \text {. }
$$

(ii)

$$
\operatorname{PKB}\left(G_{n}\right)=\sum_{u v E\left(G_{n}\right)} \frac{1}{\sqrt{S_{e}(u) S_{e}(v)}}=\frac{n}{\sqrt{n(n+1)(n+7)}}+\frac{2 n}{\sqrt{6(n+7)}}
$$


(iii)

$$
\begin{aligned}
& A B C K B\left(G_{n}\right)=\sum_{u v \in E\left(G_{n}\right)} \sqrt{\frac{S_{e}(u)+S_{e}(v)-2}{S_{e}(u) S_{e}(v)}} \\
& =n\left(\frac{n(n+1)+(n+7)-2}{n(n+1)(n+7)}\right)^{\frac{1}{2}}+2 n\left(\frac{n+7+6-2}{(n+7) 6}\right)^{\frac{1}{2}} \\
& =n\left(\frac{n^{2}+2 n+5}{n(n+1)(n+7)}\right)^{\frac{1}{2}}+2 n\left(\frac{n+11}{6(n+7)}\right)^{\frac{1}{2}} \text {. } \\
& \text { (iv) } \quad G A K B\left(G_{n}\right)=\sum_{u v \in E\left(G_{n}\right)} \frac{2 \sqrt{S_{e}(u) S_{e}(v)}}{S_{e}(u)+S_{e}(v)}=\frac{n 2 \sqrt{n(n+1)(n+7)}}{n(n+1)+n+7}+\frac{2 n 2 \sqrt{(n+7) 6}}{n+7+6} \\
& =\frac{2 n \sqrt{n(n+1)(n+7)}}{n^{2}+2 n+7}+\frac{4 n \sqrt{6(n+7)}}{n+13} \\
& R K B\left(G_{n}\right)=\sum_{u \in E\left(G_{n}\right)} \sqrt{S_{e}(u) S_{e}(v)}=n \sqrt{n(n+1)(n+7)}+2 n \sqrt{6(n+7)} . \\
& =n \sqrt{n^{2}+2 n+9}+2 n \sqrt{6(n+7)} \text {. }
\end{aligned}
$$

\section{Results for helm graphs}

A helm graph $H_{n}$ is a graph obtained from $W_{n}$ by attaching an end edge to each rim vertex. Clearly $H_{n}$ has $2 n+1$ vertices and $3 n$ edges. A graph $H_{n}$ is depicted in Figure 3.

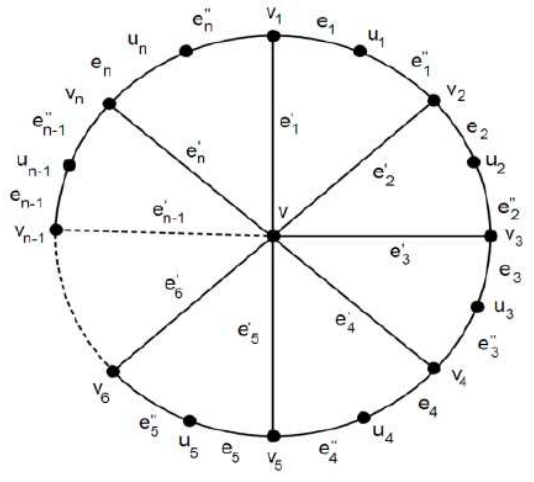

Figure 3: A graph $H_{n}$

Lemma 6. If $H_{n}$ is a helm graph with $2 n+1$ vertices and $3 n$ edges, then $H_{n}$ has three types of edges as

$$
\begin{array}{ll}
E_{1}=\left\{u v \in E\left(H_{n}\right) \mid S_{e}(u)=n(n+2), S_{e}(v)=\mathrm{n}+17\right\} & \left|E_{1}\right|=n . \\
E_{2}=\left\{u v \in E\left(H_{n}\right) \mid S_{e}(u)=S_{e}(v)=n+17\right\}, & \left|E_{2}\right|=n . \\
E_{3}=\left\{u v \in E\left(H_{n}\right) \mid S_{e}(u)=n+17, S_{e}(v)=3\right\} & \left|E_{3}\right|=n .
\end{array}
$$

Theorem 7. If $H_{n}$ is a helm graph with $2 n+1$ vertices and $3 n$ edges, then

(i) $\quad \operatorname{SKB}\left(H_{n}\right)=\frac{n}{\sqrt{n^{2}+3 n+17}}+\frac{n}{\sqrt{2 n+34}}+\frac{n}{\sqrt{n+20}}$. 
New Connectivity Topological Indices

(ii) $\quad P K B\left(H_{n}\right)=\frac{n}{\sqrt{n(n+2)(n+17)}}+\frac{n}{(n+17)}+\frac{n}{\sqrt{3(n+17)}}$.

(iii) $\quad A B C K B\left(H_{n}\right)=n\left(\frac{n^{2}+3 n+5}{n(n+2)(n+17)}\right)^{\frac{1}{2}}+n \frac{\sqrt{2 n+32}}{n+17}+n\left(\frac{n+18}{3(n+17)}\right)^{\frac{1}{2}}$.

(iv) $\operatorname{GAKB}\left(H_{n}\right)=\frac{2 n \sqrt{n(n+1)(n+7)}}{n^{2}+3 n+7}+n+\frac{2 n \sqrt{3(n+17)}}{n+20}$.

(v) $\quad R K B\left(H_{n}\right)=n \sqrt{n(n+2)(n+17)}+n(n+17)+n \sqrt{2(n+17)}$.

Proof: Let $H_{n}$ be a helm graph with $2 n+1$ vertices and $3 n$ edges. Then by using definitions and Lemma 6, we deduce

(i)

$$
\begin{aligned}
S K B\left(H_{n}\right) & =\sum_{u v \in E\left(H_{n}\right)} \frac{1}{\sqrt{S_{e}(u)+S_{e}(v)}} \\
& =\frac{n}{\sqrt{n(n+2)+n+17}}+\frac{n}{\sqrt{n+17+n+17}}+\frac{2 n}{\sqrt{n+17+3}} \\
& =\frac{n}{\sqrt{n^{2}+3 n+17}}+\frac{n}{\sqrt{2 n+34}}+\frac{n}{\sqrt{n+20}} .
\end{aligned}
$$

(ii) $\quad \operatorname{PKB}\left(H_{n}\right)=\sum_{u \in E\left(H_{n}\right)} \frac{1}{\sqrt{S_{e}(u) S_{e}(v)}}$

$$
\begin{aligned}
& =\frac{n}{\sqrt{n(n+2)(n+17)}}+\frac{n}{\sqrt{(n+17)(n+17)}}+\frac{n}{\sqrt{(n+17) 3}} \\
& =\frac{n}{\sqrt{n(n+2)(n+17)}}+\frac{n}{(n+17)}+\frac{n}{\sqrt{3(n+17)}} .
\end{aligned}
$$

(iii) $\quad A B C K B\left(H_{n}\right)=\sum_{u v E\left(H_{n}\right)} \sqrt{\frac{S_{e}(u)+S_{e}(v)-2}{S_{e}(u) S_{e}(v)}}$

$$
\begin{aligned}
& =\left|E_{1}\right| \sqrt{\frac{n(n+2)+n+17-2}{n(n+2)(n+17)}}+\left|E_{2}\right| \sqrt{\frac{n+17+n+17-2}{(n+17)(n+17)}} \\
& +\left|E_{3}\right| \sqrt{\frac{n+17+n+3-2}{(n+17) 3}} \\
& =n\left(\frac{n^{2}+3 n+15}{n(n+2)(n+17)}\right)^{\frac{1}{2}}+n \frac{\sqrt{2 n+32}}{n+17}+n\left(\frac{n+18}{3(n+7)}\right)^{\frac{1}{2}} .
\end{aligned}
$$

(iv) $\quad \operatorname{GAKB}\left(H_{n}\right)=\sum_{u v E\left(H_{n}\right)} \frac{2 \sqrt{S_{e}(u) S_{e}(v)}}{S_{e}(u)+S_{e}(v)}$

$$
=\left|E_{1}\right| \frac{2 \sqrt{n(n+2)(n+17)}}{n(n+2)+n+17}+\left|E_{2}\right| \frac{2 \sqrt{(n+17)(n+17)}}{n+17+n+17}
$$




$$
\begin{aligned}
& +\left|E_{3}\right| \frac{2 \sqrt{(n+17) 3}}{n+17+3} \\
= & \frac{2 n \sqrt{n(n+2)(n+7)}}{n^{2}+3 n+17}+n+\frac{2 n \sqrt{3(n+17)}}{n+20} .
\end{aligned}
$$

(v)

$$
\begin{aligned}
\operatorname{RKB}\left(H_{n}\right) & =\sum_{u \in E\left(H_{n}\right)} \sqrt{S_{e}(u) S_{e}(v)} \\
& =\left|E_{1}\right| \sqrt{n(n+2)(n+17)}+\left|E_{2}\right| \sqrt{(n+17)(n+17)}+\left|E_{3}\right| \sqrt{(n+17) 3} . \\
& =n \sqrt{n(n+2)(n+17)}+n(n+17)+n \sqrt{3(n+17)} .
\end{aligned}
$$

Acknowledgement. The author is thankful to the referee for his/her suggestions.

\section{REFERENCES}

1. V.R.Kulli, College Graph Theory, Vishwa International Publications, Gulbarga, India (2012).

2. I.Gutman and O.E.Polansky, Mathematical Concepts in Organic Chemistry, Springer, Berlin (1986).

3. V.R.Kulli, Multiplicative Connectivity Indices of Nanostructures, LAP LEMBERT Academic Publishing (2018).

4. B.Basavanagoud, P.Jakkannavar, Kulli-Basava indices of graphs, Inter. J. Appl. Engg. Research, 14(1) (2018) 325-342.

5. V.R.Kulli, Some new topological indices of graphs, International Journal of Mathematical Archive, 10(5) (2019) 62-70.

6. V.R.Kulli, Product connectivity leap index and $A B C$ leap index of helm graphs, Annals of Pure and Applied Mathematics, 18(2) (2018) 189-193.

7. V.R.Kulli, The sum connectivity Revan index of silicate and hexagonal networks, Annals of Pure and Applied Mathematics, 14(3) (2017) 401-406.

8. V.R.Kulli, Computing reduced connectivity indices of certain nanotubes, Journal of Chemistry and Chemical Sciences, 8(11) (2018) 1174-1180.

9. V.R.Kulli, Degree based connectivity $F$-indices of dendrimers, Annals of Pure and Applied Mathematics, 18(2) (2018) 201-206.

10. V.R.Kulli and M.H.Akhabari, Multiplicative atom bond connectivity and multiplicative geometric-arithmetic indices of dendrimer nanostars, Annals of Pure and Applied Mathematics, 16(2) (2018) 429-436. 